\title{
Distance determination to six nearby galaxies using type IIP supernovae.
}

\author{
Subhash Bose and Brijesh Kumar \\ Aryabhatta Research Institute of Observational Sciences, Nainital, India \\ email: bose@aries.res.in; email@subhashbose.com
}

\begin{abstract}
We use early optical photometric and spectroscopic data of six Type-IIP SNe to derive distances to their host galaxies using the expanding photosphere method (EPM). Our sample consists of luminous to sub-luminous SNe 1999gi, 2004et, 2005cs, 2008in, 2009md and 2012aw; having absolute V-magnitudes from -17 to -15 and host galaxy distances from 5 to $22 \mathrm{Mpc}$. The SN 2008in is peculiar in nature showing dual behavior of a luminous as well as sub-luminous event. The EPM distances for four of the events in our sample are derived for the first time. We take utmost care in minimizing the errors arising from photospheric velocity determination and the broadband filter responses, hence leaving out uncertainty in dilution factor models as the only major source of error. Our preliminary results indicate that EPMderived distances using Dessart model is found to be consistent with the distances quoted in the literature. We find that EPM method is applicable only to the early $(<50 \mathrm{~d})$ photometric data of supernovae and dense spectroscopic data is necessary to estimate accurate distances.
\end{abstract}

Keywords. galaxies: distances and redshifts, (stars:) supernovae: general,(stars:) supernovae: individual (2012aw, 2004et, 2005cs, 2008in, 2009md, 1999gi)

\section{Introduction}

Supernovae owing to their high luminosity, are the objects of interest for extragalactic distance estimation. Type II supernovae are not used traditionally as standard candles for distance measurement. However, in case of type II-P SNe, assuming spherically symmetric expansion of their ejecta and radiating isotropically as a blackbody at a well defined temperature, we can apply the Expanding Photosphere Method (EPM; Kirshner \& Kwan 1974) a variant of Baade-Wesselink method to determine distances to their host galaxies.

\subsection{Expanding Photosphere Method}

The EPM is fundamentally a geometrical technique, in which we compare the linear radii determined from the expansion velocity and angular radii of the supernova by fitting blackbody with the observed fluxes at different epochs.

Assuming homologous expansion, we may relate photospheric velocity $v_{\text {phot }}$, angular radius $\theta$ and distance $D$ at time $t$ and $t_{0}$ be the explosion epoch, we may write

$$
\begin{array}{r}
\quad \theta=\frac{v_{p h o t}\left(t-t_{0}\right)}{D} \\
t=D\left(\frac{\theta}{v_{\text {phot }}}\right)+t_{0}
\end{array}
$$

Hence we get a linear equation, whose slope yields the distance to $\mathrm{SN}(D)$ and the $y$-intercept as the explosion epoch $\left(t_{0}\right)$.

To determine $\theta$ we assume SN radiating isotropically as blackbody and accounting for the conservation of radiative energy we may write,

$$
4 \pi R^{2} \cdot \pi B_{\lambda}\left(T_{c}\right)=4 \pi D^{2} f_{\lambda}^{\text {dered }}
$$


where $B_{\lambda}\left(T_{c}\right)$ is Planck Blackbody function at color temperature $T_{c}$ and $f_{\lambda}^{\text {dered }}$ is the extinction corrected (de-reddened) observed flux. Absorbing the $R$ and $D$ into $\theta$ we may write,

$$
f_{\lambda}^{\text {dered }}=\theta^{2} \pi B_{\lambda}\left(T_{c}\right)
$$

Now introducing the wavelength dependent extinction $A_{\lambda}$ and writing in terms of observed flux $f_{\lambda}$,

$$
f_{\lambda}=\theta^{2} \pi B_{\lambda}\left(T_{c}\right) 10^{-0.4 A_{\lambda}}
$$

On minimizing the above equation with two or more known observed fluxes $f_{\lambda}^{o b s}$ and the Planckian blackbody model function we can determine the angular radius $\theta$ and the color temperature $T_{c}$ both together. Thus to derive distance by EPM, all we need are observed flux $f_{\lambda}$ and photospheric velocity $v_{\text {phot }}$.

One of the most important assumption that goes into EPM, is to consider the expanding photosphere radiating as blackbody. There might be a significant departure from a blackbody atmosphere and this will be directly reflected into the distance estimated by EPM. The thermalization layer from which the thermal photons are generated is significantly deeper than photospheric layer from which photons start to flow freely without any further scattering (i.e. the surface of last scattering at $\tau=\frac{2}{3}$ ), to take care of this discrepancy, we use "dilution factor" $\xi$ as

$$
\xi=\frac{R_{\text {therm }}}{R_{\text {phot }}}
$$

and rewrite the equation (1.4) as,

$$
f_{\lambda}=\xi^{2} \theta^{2} \pi B_{\lambda}\left(T_{c}\right) 10^{-0.4 A_{\lambda}}
$$

In principle this factor should depend upon many physical properties including chemical properties, density profile of the SNe etc. However, studies have shown (Eastman et al. $1996)$ that $\xi$ more or less behaves as one-dimensional function of color temperature, $T_{c}$ only. The computation of $\xi$ requires realistic SN atmosphere models. Till date, two prescription for dilution factors are available, Hamuy et al. (2001) (hereafter H01) which is improved estimate of $\xi$ over Eastman et al. (1996) used 63 stellar atmospheric models, whereas the other Dessart \& Hillier (2005) (hereafter D05) which uses CMFGEN models for $\mathrm{SN}$ to determine $\xi$ for different filter pass-band combinations.

In order to improve the accuracy of our study, we used SYNOW (Branch et al. 2001, Branch et al. 2001, Elmhamdi et al. 2006) to model the spectra for each of the event to determine their photospheric velocities $v_{p h o t}$ at different phases. Further to remove the effect of filter response which is intrinsically embedded in observed flux, we convolve the response function $\Re_{\lambda}\left(\lambda^{\prime}\right)$ for each pass-band filter with the blackbody model to obtain the synthetic model flux. be the normalized response function of a particular filter whose effective wavelength is $\lambda$, then the convolved synthetic flux $b_{\lambda}$ is,

$$
b_{\lambda}\left(T_{c}\right)=\int_{0}^{\infty} \Re_{\lambda}\left(\lambda^{\prime}\right) \pi B\left(\lambda^{\prime}, T_{c}\right) d \lambda^{\prime}
$$

Hence in the equation 1.6, the blackbody flux is replaced with convolved blackbody flux $b_{\lambda}$ for each filter and rewritten as,

$$
f_{\lambda}=\xi^{2} \theta^{2} b_{\lambda}\left(T_{c}\right) 10^{-0.4 A_{\lambda}}
$$


Table 1. Adopted parameters

\begin{tabular}{|l|l|r|r|}
\hline SN & $E(B-V)$ & $\begin{array}{r}\text { Reference epoch } \\
(J D)\end{array}$ & $\begin{array}{r}\text { Recession Velocity } \\
\left(\mathrm{km} \mathrm{s}^{-1}\right)\end{array}$ \\
\hline SN1999gi & 0.21 & $2451518.3 \pm 3.1$ & 552 \\
SN2004et & 0.41 & $2453270.5 \pm 0.9$ & 45 \\
SN2005cs & 0.05 & $2453549.0 \pm 1.0$ & 463 \\
SN2008in & 0.098 & $2454825.6 \pm 2.0$ & 1567 \\
SN2009md & 0.1 & $2455162.0 \pm 8.0$ & 1308 \\
SN2012aw & 0.075 & $2456002.6 \pm 0.8$ & 778 \\
\hline
\end{tabular}

Table 2. Table of derived EPM distances to host galaxies and comparison with NED result.

\begin{tabular}{|c|c|c|c|c|}
\hline Host galaxy & SN event & $\begin{array}{c}\text { EPM Distance }^{a} \\
\text { Mpc }\end{array}$ & Distance Modulus & $\begin{array}{c}\text { NED Distance } \\
\text { Mpc }\end{array}$ \\
\hline NGC 3351/M95 & SN 2012aw & $9.83 \pm 0.41$ & $29.96 \pm 0.09$ & $10.11 \pm 0.98$ \\
\hline NGC 6946 & SN 2004et & $5.86 \pm 0.76$ & $28.84 \pm 0.28$ & $5.96 \pm 1.97$ \\
\hline NGC 3184 & SN 1999gi & $11.62 \pm 0.29$ & $30.33 \pm 0.05$ & $11.95 \pm 2.71$ \\
\hline NGC 4303/M61 & SN 2008in & $14.51 \pm 1.38$ & $30.81 \pm 0.21$ & $16.46 \pm 10.84$ \\
\hline NGC 5194/M51a & SN $2005 \mathrm{cs}$ & $8.01 \pm 0.62$ & $29.52 \pm 0.17$ & $7.91 \pm 0.87$ \\
\hline NGC 3389 & SN 2009md & $23.29 \pm 1.96$ & $31.84 \pm 0.18$ & $21.29 \pm 2.21$ \\
\hline
\end{tabular}

${ }^{a}$ EPM distances are using D05 prescription.

${ }^{b}$ NED (http://ned.ipac.caltech.edu/) distance is mean value of all redshift independent distances listed in NED for the galaxy. Errors are the STD DEV of the listed distances.

\section{Sample selection and data}

For the EPM study we select a sample of six recent II-P SNe viz., 1999gi (Leonard et al. 2002), 2004et (Sahu et al. 2006), 2005cs (Pastorello et al. 2006, Pastorello et al. 2009), 2008in (Roy et al. 2011), 2009md (Fraser at al. 2011) and a very recent event 2012aw (in preparation Bose et al.); which comprises of normal to sub-luminous type events having good photometric and spectroscopic follow-up including early plateau phase. Among these, 1999gi, 2004et and 2012aw are normal events; 2005cs and 2009md are sub-luminous whereas 2008in lies in between normal and sub-luminous II-P events. Th adopted parameters from literature are tabulated in Table $1, E(B-V)$ are used to de-redden corresponding photometric and spectroscopic data, Recession velocity are used to doppler correct spectra and reference epoch are the explosion epochs adopted in corresponding literature, in reference to these epochs we independently estimate explosion epochs from EPM.

\section{Preliminary Results}

EPM is applied to each of these six events and corresponding distance and explosion epoch are determined for each set of dilution factor models viz., D05 and H01 with each of three filters subsets $B V, B V I$ and $V I$. For the SNe 2012aw, 2005cs and 2004et, EPM is also applied by fixing explosion epochs to their observationally constrained explosion epochs having accuracy less than a day and thus keeping Distance as the only free parameter for EPM fit. Figure 1 is the EPM fit for SN 2012aw with both explosion epoch and distance, while the Figure 2 is the EPM fit with fixed explosion epoch having distance as only free parameter. Similar EPM fits are done for all other events and 


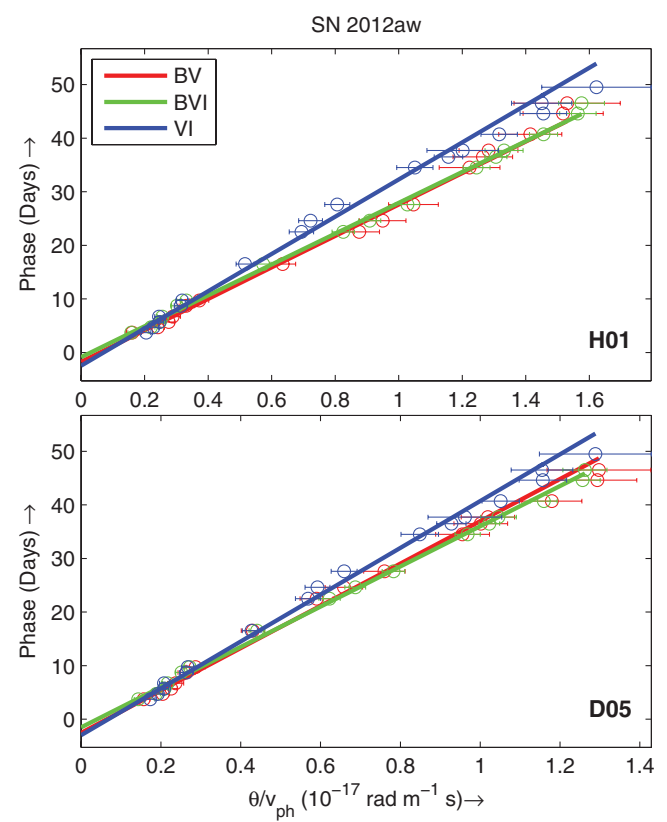

Figure 1. EPM fitting for SN 2012aw using both D05 and H01 prescriptions.
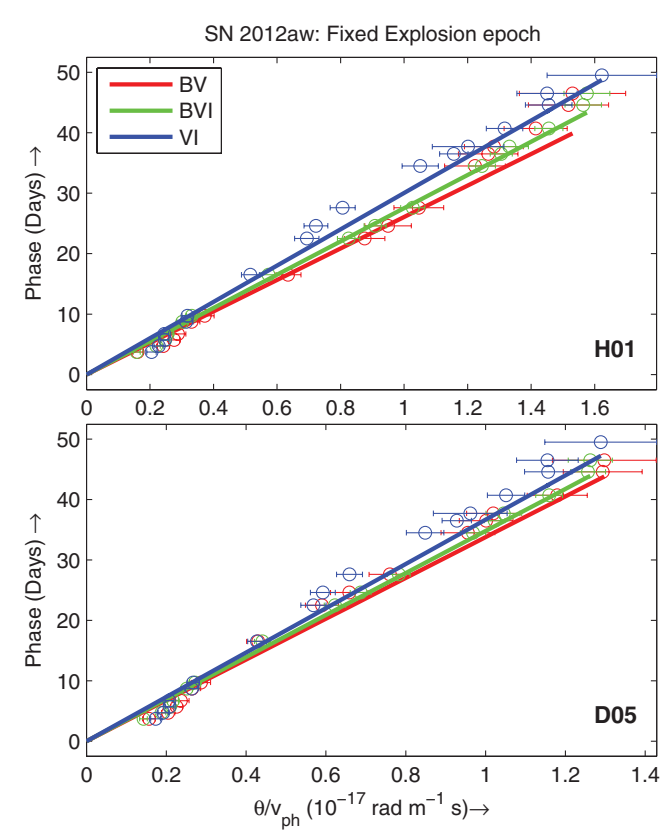

Figure 2. EPM fitting for SN 2012aw using both D05 and H01 prescriptions with fixed explosion epoch and Distance as the only free parameter.

EPM determined distances using D05 prescription are tabulated in Table 2 (a detailed analysis is in preparation, Bose et al.). The EPM distances using D05 are found to be more consistent than those by H01 with NED listed distances for corresponding galaxies.

\section{Conclusion}

The D05 prescription of dilution factors are found to be more suitable and accurate for EPM analysis. Also the SYNOW derived photospheric velocities significantly improve the accuracy of distance determination.

\section{References}

Branch, D., Baron, E., \& Jeffery, D. J. 2001, arXiv:astro-ph/0111573

Branch, D., Benetti, S., Kasen, D., et al. 2002, ApJ, 566, 1005

Dessart, L. \& Hillier, D. J. 2005, A\&A, 439, 671

Eastman, R. G., Schmidt, B. P., \& Kirshner, R. 1996, ApJ, 466, 911

Elmhamdi, A., Danziger, I. J., Branch, D., et al. 2006, A\& $A, 450,305$

Fraser, M., Ergon, M., Eldridge, J. J., et al. 2011, MNRAS, 417, 1417

Hamuy, M., Pinto, P. A., Maza, J., et al. 2001, ApJ, 558, 615

Kirshner, R. P. \& Kwan, J. 1974, ApJ, 193, 27

Leonard, D. C., Filippenko, A. V., Li, W., et al. 2002, AJ, 124, 2490

Pastorello, A., Sauer, D., Taubenberger, S., et al. 2006, MNRAS, 370, 1752

Pastorello, A., Valenti, S., Zampieri, L., et al. 2009, MNRAS, 394, 2266

Roy, R., Kumar, B., Benetti, S., et al. 2011, ApJ, 736, 76

Sahu, D. K., Anupama, G. C., Srividya, S., \& Muneer, S. 2006, MNRAS, 372, 1315 


\section{Discussion}

Bersten: Have you compared your results of distance and tpxp with the paper of Jones et al. 2010. In this paper they used the same atmospheric models that you use in this work (DO5)? It would be very interesting to know how the results compare.

Bose: No, we haven't compared with this particular paper, however, the mean of redshift independent distances from NED, with which we compare our result, some has SEAM distances included also. Moreover SEAM and EPM are quite different in terms of technique used, in SEAM, dilution factors are not used separately, since the SED used in this case is not LTE approximation, the SED used in SEAM, itself taken into account the non-LTE atmosphere of SN. However the comparison of these two methods will be interesting.

Follatelli: Have you computed Ho based on your distances?

Bose: Our sample is quite small. Only comprising of 6 events, hence it cannot be used to construct Hubble diagram. Furthermore, 5 of the events are nearby $(<15 \mathrm{Mpc})$. Thus it is not suitable to compute $\mathrm{H} 0$ from this sample. However, in future more EPM based new studies will be added to this sample and may be used to compute H0. 